\title{
ESTRUTURA ESPACIAL DE TRÊS ESPÉCIES ARBÓREAS RELACIONADAS AO SETOR ALIMENTÍCIO
}

\author{
Maurício Koubay do Amaral ${ }^{1}$, Sylvio Péllico Netto ${ }^{2}$, Christel Lingnau ${ }^{2}$, Vagner Alex Pesk ${ }^{3}$, \\ Rorai Pereira Martins Neto ${ }^{4}$
}

\begin{abstract}
${ }^{1}$ Universidade Tecnológica Federal do Paraná, Departamento de Matemática, Curitiba, Paraná, Brasil - amaral@utfpr.edu.br ${ }^{2}$ Universidade Federal do Paraná, Departamento de Ciências Florestais, Curitiba, Paraná, Brasil - sylviopelliconetto@ gmail.com; clingnau@yahoo.com.br

${ }^{3}$ Universidade Estadual do Centro-Oeste, Departamento de Engenharia Florestal, Irati, Paraná, Brasil - vagneralexp@ yahoo.com.br ${ }^{4}$ Universidade do Estado de Santa Catarina, Programa de Pós-Graduação em Engenharia Florestal, Lages, Santa Catarina, Brasil rorai.neto@gmail.com
\end{abstract}

Recebido para publicação: 04/10/2013 - Aceito para publicação: 28/05/2014

\begin{abstract}
Resumo
Este trabalho trata da estrutura espacial e da modelagem da distribuição diamétrica de três espécies com potencial comercial para produtos não madeireiros. Os dados foram coletados em um fragmento de Floresta Ombrófila Mista, localizado no segundo planalto paranaense, entre os municípios de Fernandes Pinheiro e Teixeira Soares, a $5 \mathrm{~km}$ de Irati, PR. A pesquisa apresenta o padrão de dispersão espacial das espécies e o ajuste das distribuições de diâmetro, utilizando as funções densidade de probabilidade Beta, Gama e Weibull-3P. As discussões mostraram que as espécies têm a mesma classificação de dispersão agregada, mas com grande diferença na amplitude dos resultados, motivo pelo qual foi necessário elaborar um layout das espécies, para que elas pudessem ser mais bem compreendidas em seus arranjos espaciais, o que permite visualizar a forma mais apropriada de acesso a esses indivíduos. A FDP Weibull-3P foi a que melhor se ajustou aos dados das distribuições diamétricas. A relação entre a disposição espacial dos indivíduos e as curvas estimadas pelas distribuições diamétricas permitem substanciar melhor a elaboração do planejamento estratégico para o manejo florestal da área, em que espécies fornecedoras de produtos não madeireiros se destacam como opção complementar à utilização de madeira para fins comerciais.

Palavras-chave: Índice de Morisita; produtos não madeiráveis; distribuição diamétrica.
\end{abstract}

\begin{abstract}
Spatial structure and modeling diametric distributions of three tree species related to food sector. This paper deals with the spatial structure and modeling the diameter distribution of three species with potential inclusion of commercial non-timber products. Data from Araucaria Forest were collected in a fragment located on the second plateau of Parana between the municipalities of Fernandes Pinheiro and Teixeira Soares, $5 \mathrm{~km}$ from Irati, PR. The research presents the dispersion pattern of the species and adjustment of diameter distributions Beta, Gama and Weibull-3P. The discussions revealed that the species have the same classification of aggregate dispersion, but great difference in the amplitude of results, so it was necessary drawing a layout of the species, in order to better comprehension of their spatial arrangements, it allows us to visualize the better way of access to these individuals. The fdp Weibull-3P is the distribution that fits better to the data of diameter distributions. The relationship between the spatial distribution of individuals and the curves estimated by the diameter distributions allow substantiating the strategic planning for the forest management area, where suppliers of non-timber products stand out as complementary option to the use of timber for commercial purposes.
\end{abstract}

Keywords: Morisita index; non-timber products; diameter distributions.

\section{INTRODUÇÃO}

A exploração ou colheita sustentável de produtos não madeireiros requer controle e monitoramento periódico da regeneração e crescimento das espécies, ou seja, necessita de uma maior 
valorização de aspectos ecológicos e de manejo florestal, pois são os pilares dessa exploração sustentável dos recursos (PETERS, 1994). No que concerne isso, atentou-se para a realização de uma pesquisa que mostrasse a importância do estudo do comportamento espacial na disposição dos indivíduos aliado à modelagem dos diâmetros na fundamentação de um planejamento estratégico para colheita de produtos não madeireiros.

Para isso, foram adotados como elementos da investigação três espécies arbóreas da Floresta Ombrófila Mista (FOM), que se destacam pela importância dos seus produtos não madeiráveis. A exploração delas é pouco realizada, causada, muitas vezes, pela falta de estudos relacionados à modelagem do seu comportamento biométrico e de sua estrutura espacial. Uma dessas espécies é Eugenia involucrata, que possui frutos próprios para consumo humano e animal e também flor com substâncias melíferas. No Brasil, essa espécie é amplamente cultivada em pomares domésticos em toda a região Sul, principalmente entre os colonos teuto-brasileiros (ORO et al., 2012). A outra espécie é Myrciaria cauliflora, que apresenta frutos para serem consumidos in natura ou na forma de geleias, sucos e licores, entre outras, servindo também de alimento para a fauna (DONADIO, 2000). Possui grande potencial ornamental e crescimento lento, demandando de 10 a 20 anos para a primeira frutificação (SASSO, 2009). Depois, sua produção é contínua, sendo uma das espécies mais cultivadas em pomares domésticos. A terceira espécie é llex paraguariensis, cujas folhas são muito utilizadas pelas suas características estimulantes, digestivas e antioxidantes, muito cultivada em pequenas propriedades no sul do país e no Mato Grosso do Sul, garantindo uma posição de importância social (WENDT et al., 2007). As folhas são consumidas, em sua maioria, na forma de bebidas, como chás, chimarrão e tererê (MACCARI JÚNIOR, 2000).

A primeira análise deste estudo ocorreu por meio da classificação do comportamento na disposição espacial dos indivíduos, que é de grande interesse para o manejo florestal, uma vez que está relacionada ao crescimento dos indivíduos, à distribuição diamétrica, à densidade de árvores e, consequentemente, à produção volumétrica (PUKKALA, 1988 e 1989; LÖETSCH et al., 1973). Ela tem sido efetuada por meio de índices, dos quais destacam-se o índice de Morisita e a razão variância pela média, que informam, por meio dos seus resultados, o conhecimento do arranjo de indivíduos na área amostral. Segundo Barbosa (1992), esse arranjo é classificado como agregado (ou contagioso), aleatório (ou ao acaso) e uniforme (ou regular), no entanto o grau ou o nível dessa disposição não fica evidenciado com clareza, pois faltam informações no que se refere à alocação ou distribuição na área que está sendo investigada.

O segundo processo está relacionado à descrição da estrutura diamétrica de um povoamento, que é efetuada com aplicação das funções densidade de probabilidade (FDP), que facilitam o planejamento da produção da floresta (BARRA et al., 2004). A distribuição dos diâmetros é um procedimento biométrico de fácil obtenção e eficiente para a caracterização de um povoamento, sendo um instrumento importante para o controle das atividades florestais desenvolvidas, tendo em vista que possibilita o conhecimento de produções futuras, número de árvores e volume por hectare para cada classe de diâmetro (CARELLI NETTO; SCHNEIDER, 2008; MACHADO et al., 2009). Existem vários tipos de distribuição que podem descrever a estrutura diamétrica de um povoamento. As de maior destaque são: distribuição Gama (NELSON, 1964), Beta (CLUTTER; BENNETT, 1965) e Weibull (BAILEY; DELL, 1973). Os parâmetros dessas funções são estimados por diferentes métodos, tais como Momentos, Regressão, Máxima Verossimilhança e Percentis, além de outros estimadores que têm sido desenvolvidos por inúmeros pesquisadores nos últimos anos (ORELLANA, 2009).

Trabalhos relativos a esses dois processos (comportamento espacial versus modelagem matemática) foram estudados por diversos autores (FARIAS et al., 2001; MARUYAMA et al., 2006), trabalhando em pesquisas relacionadas às espécies de insetos causadoras de doenças sobre algum tipo de cultivo, porém com objetivos semelhantes aos deste trabalho no que concerne às espécies arbóreas mencionadas acima, como a otimização de técnicas de amostragem e a incorporação da dinâmica espacial.

Souza e Silva (2006) defenderam o uso do processo da análise do comportamento na disposição dos indivíduos como instrumento do conhecimento da estrutura espacial das espécies, relatando que isso é necessário para o entendimento de sua ecologia e que serve como ferramenta para avaliar se as populações estão estáveis ou se estão sendo substituídas por outras, além de estabelecer diferenças entre impactos antrópicos e processos dinâmicos naturais. 
Assim, este trabalho teve como objetivo mostrar a importância da relação entre o comportamento espacial na disposição dos indivíduos e da modelagem diamétrica das espécies como informações necessárias em um planejamento estratégico de manejo e/ou conservação florestal para espécies com potencial comercial no âmbito dos produtos do setor alimentício.

\section{MATERIAIS E MÉTODOS}

A pesquisa foi realizada com dados do censo de 2007/2008 de três espécies arbóreas - Eugenia involucrata (cerejeira-do-mato), Myrciaria cauliflora (jabuticabeira) e Ilex paraguariensis (erva-mate) -, oriundas de um fragmento de Floresta Ombrófila Mista (FOM), situada no segundo planalto paranaense, entre os municípios de Fernandes Pinheiro e Teixeira Soares, a $5 \mathrm{~km}$ do município de Irati, estado do Paraná. Essa floresta de onde foram retirados os dados das espécies tem em sua estrutura a ocorrência da mistura de floras de diferentes origens, definindo padrões fitofisionômicos típicos, em zonas climáticas caracteristicamente pluviais. A Floresta Nacional (FLONA) de Irati faz parte dessa formação florestal, onde foram instaladas, em 2002, 25 parcelas permanentes de um hectare $(100 \times 100 \mathrm{~m})$ cada uma. Cada parcela foi subdividida em quatro subparcelas de 0,25 hectares $(50 \times 50 \mathrm{~m})$ (Figura 1).

Naquele mesmo ano, todas as árvores com DAP igual ou maior que $10 \mathrm{~cm}$ foram medidas, identificadas, numeradas e posicionadas em um sistema cartesiano (x,y), sendo que, nos anos de 2005, 2008 e 2011, todas as árvores foram remedidas, a fim de avaliar a dinâmica da floresta (DISPERATI, 2002, 2006).

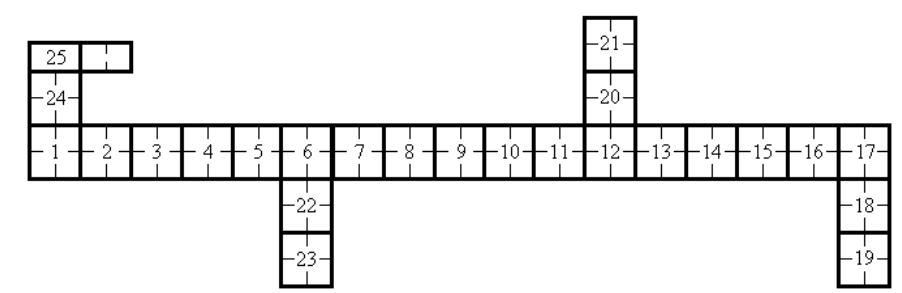

Figura 1. Layout dos blocos (1 ha) e das parcelas ( 0,25 ha).

Figure 1. Layout of the blocks (1 ha) and plots ( $0.25 \mathrm{ha})$.

Rode (2008 e 2010) descreveu minuciosamente essa floresta, utilizando dados advindos da medição/remedição de 2004-2005 sobre avaliação florística e estrutural, constatando que as espécies mais presentes na área eram Ilex paraguariensis (erva-mate), Ocotea odorifera (sassafrás) e Araucaria angustifolia (araucária), que apresentaram em média 55, 52 e 42 indivíduos/ha, respectivamente. Também numerou e catalogou 14.010 indivíduos $\left(560,4\right.$ ind.ha $\left.^{-1}\right)$ de 72 gêneros e 108 espécies, além de determinar, por meio do índice de Shannon $\left(\mathrm{H}^{\prime}=3,55\right)$, a diversidade florística da vegetação estabelecida sob a floresta natural. Isso mostra que as espécies selecionadas sobrevivem em grupamentos que não são contínuos nem homogêneos, e sim formados por muitas associações e aglomerações, dispersos em diversos estágios de sucessão.

O delineamento da pesquisa foi estruturado com a classificação do padrão de dispersão espacial e da modelagem das distribuições diamétricas dos indivíduos, por meio do ajuste de três funções densidade de probabilidade. Essa classificação se deu por meio da aplicação da Equação (1) do índice de Morisita (MORISITA, 1962) aos dados oriundos da coleta de amostragem aplicada a todos os indivíduos dessas três espécies que foram retirados das parcelas permanentes.

$$
I_{\delta}=q\left(\frac{\sum_{i=1}^{q} x^{2}-\sum_{i=1}^{q} x}{\left(\sum_{i=1}^{q} x\right)^{2}-\sum_{i=1}^{q} x}\right)
$$

em que: $q=$ número total de subparcelas na área amostral;

$\sum_{i=1}^{q} x=$ soma dos indivíduos da mesma espécie em cada subparcela na área amostral;

$\sum_{i=1}^{q} x^{2}=$ soma do quadrado dos indivíduos da mesma espécie em cada subparcela na área amostral. 
Para aplicação do processo de junção desses dois métodos, foram selecionadas três espécies arbóreas com características frutícolas e aromáticas, além do apreço de seus produtos pelo mercado alimentício: Eugenia involucrata DC. (cerejeira-do-mato), Myrciaria cauliflora O. Berg. (jabuticabeira) e Ilex paraguariensis St.-Hil. (erva-mate).

Os resultados dessa classificação seguiram a definição de Nascimento (1995), que explicita: valores próximos ao valor um indicam arranjo ao acaso; valores superiores a um indicam disposição agregada; e valores inferiores a um indicam arranjo uniforme.

Para análise comparativa desses resultados com a disposição espacial dos indivíduos, foi necessário configurar a sua espacialização, utilizando-se suas coordenadas locais (x,y) como identificadores da posição de cada indivíduo, a fim de verificar se os resultados do índice de Morisita estavam em harmonia com a estrutura de disposição dos indivíduos na área amostrada.

A modelagem do crescimento diamétrico das espécies ocorreu por meio do ajuste de três funções densidade de probabilidade, escolhidas por sua alta aplicabilidade em pesquisas florestais. As funções Beta, Gama e Weibull 3 foram ajustadas pelo método da máxima verossimilhança, e a descrição dessas funções, juntamente com os estimadores da média e da variância, estão exibidos na tabela 1.

Tabela 1. Função densidade de probabilidade das distribuições Beta, Gama e Weibull.

Table 1. Probability density function of distributions Beta, Gamma and Weibull.

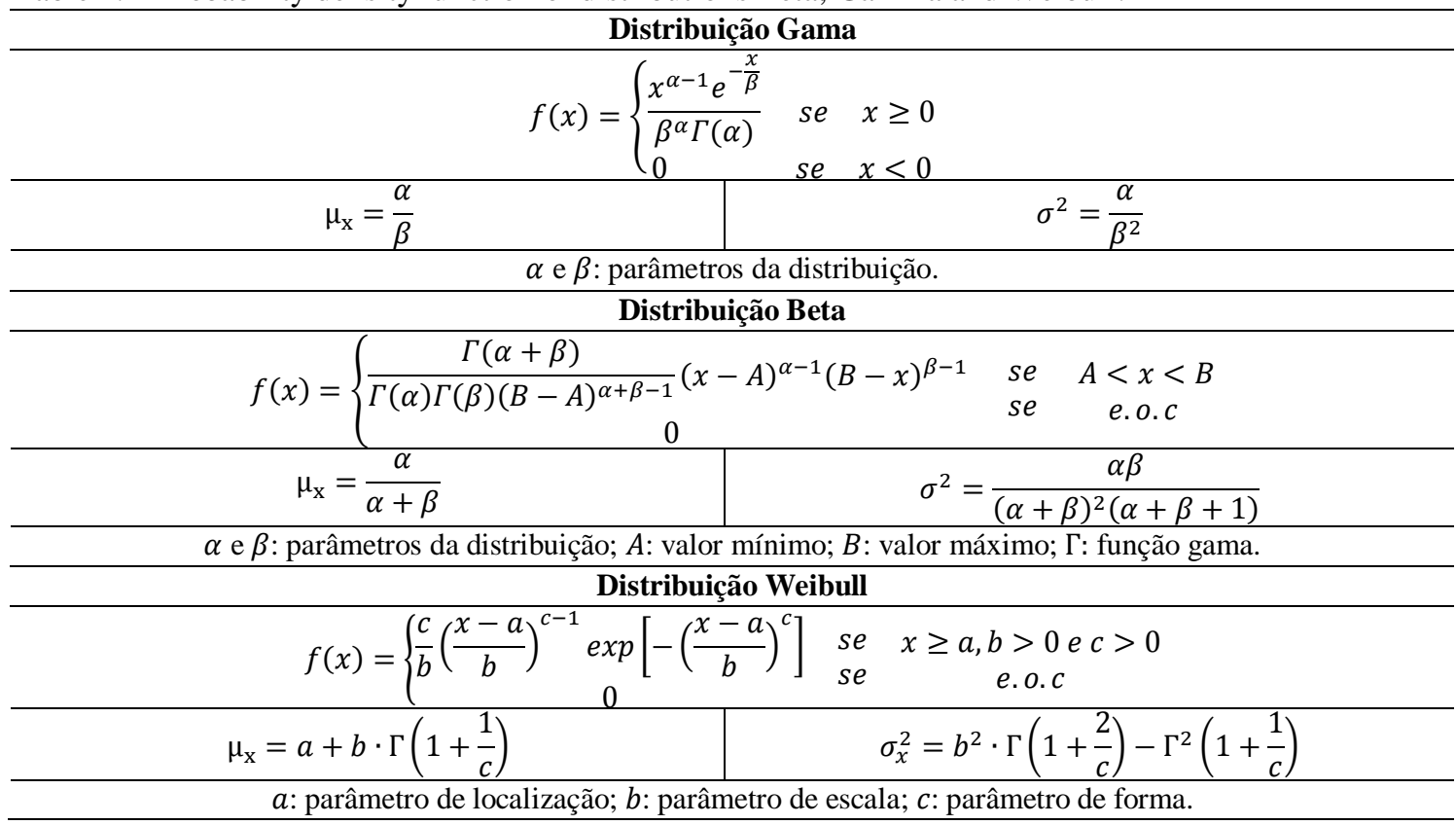

Para verificar a aderência das funções de distribuição, foi aplicado o teste de KolmogorovSmirnov (K-S), com nível de significância de 5\%, utilizado para avaliar se as distribuições ajustadas se adequam bem aos dados dos diâmetros agrupados em classes de frequência. Já a verificação da adequação dos ajustes e precisão dos dados estimados ocorreu por meio do coeficiente de determinação $\left(R^{2}\right)$ e do erro padrão da estimativa $\left(S_{\mathrm{yx}}\right)$, além da análise gráfica de resíduos.

A técnica utilizada na coleta dos dados foi o censo, ou seja, a medição de todos os indivíduos das três espécies selecionadas com diâmetro igual ou maior que $10 \mathrm{~cm}$ das 100 subparcelas dos 25 hectares. Isso foi realizado entre os anos de 2007/2008, com as seguintes variáveis medidas: número de indivíduos da mesma espécie nas subparcelas, número de subparcelas com indivíduos da mesma espécie, diâmetro (DAP) e registro das coordenadas locais (x,y) dos indivíduos.

Por fim, ocorreu a análise da relação gráfica dos dados de crescimento dos diâmetros das espécies com os resultados obtidos por meio da aplicação do índice de Morisita. 


\section{RESULTADOS E DISCUSSÃO}

A tabela 2 mostra que as três espécies são classificadas como agregadas pelo índice de Morisita, porém percebe-se, por meio dos valores das variáveis de campo (NIS e NSI), que existem diferenças nos dados e, consequentemente, no grau de classificação das espécies. Entretanto, não é possível identificar o comportamento espacial na disposição dos indivíduos, sendo esse fato relevante na construção de um planejamento estratégico para a exploração dos seus produtos.

Tabela 2. Classificação do padrão de dispersão espacial das três espécies pelo índice de Morisita.

Table 2. Classification of the spatial dispersion pattern of three species by Morisita index.

\begin{tabular}{|c|c|c|c|c|c|}
\hline \multirow{2}{*}{$\begin{array}{l}\text { Padrão } \\
\text { espacial }\end{array}$} & \multicolumn{2}{|c|}{ Espécies } & \multirow{2}{*}{ NIS } & \multirow{2}{*}{ NSI } & \multirow{2}{*}{$\begin{array}{l}\text { Resultado do índice } \\
\text { de Morisita }\left(I_{\delta}\right)\end{array}$} \\
\hline & Nome científico & Nome vulgar & & & \\
\hline \multirow{3}{*}{ Agregado } & Myrciaria cauliflora & jabuticabeira & 37 & 19 & 5,11 \\
\hline & Eugenia involucrata & cerejeira & 206 & 82 & 1,54 \\
\hline & Ilex paraguariensis & erva-mate & 1334 & 100 & 1,13 \\
\hline
\end{tabular}

NIS: número de indivíduos da mesma espécie nas subparcelas; NSI: número de subparcelas com indivíduos da mesma espécie.

A falta desse conhecimento induz a pesquisa ao uso das coordenadas cartesianas locais $(\mathrm{x}, \mathrm{y})$, as quais resultam na espacialização dos seus indivíduos (Figura 2) e, assim, mostra suas reais disposições nas parcelas permanentes, servindo como instrumento teórico na comparação entre o grau de agregação e o resultado do índice de Morisita, assim como ponto de partida para o desenvolvimento do planejamento (Tabela 2).

A espécie Myrciaria cauliflora é a mais agregada entre as três, segundo o resultado do índice de Morisita (Tabela 2), porém nota-se, na figura 2, que essa espécie possui poucos indivíduos na área amostral quando comparada às demais, o que indica impropriedade de sua inclusão no manejo florestal, pois o processo exploratório do fruto jabuticaba se tornaria não vantajoso, devido a seu custo/benefício ser desinteressante nessas condições. Ainda, sobre essa espécie, existe a informação (Figura 2) de sua disposição na forma agregada em 11 subparcelas, que fazem parte dos blocos 5, 6, 7 e 8 (Figura 1). Essa informação também é importante para o deslocamento a ser percorrido, caso seja definido introduzir o processo de colheita dos frutos, ou para uma análise mais detalhada sobre os indivíduos, como porte, sanidade e forma da copa.
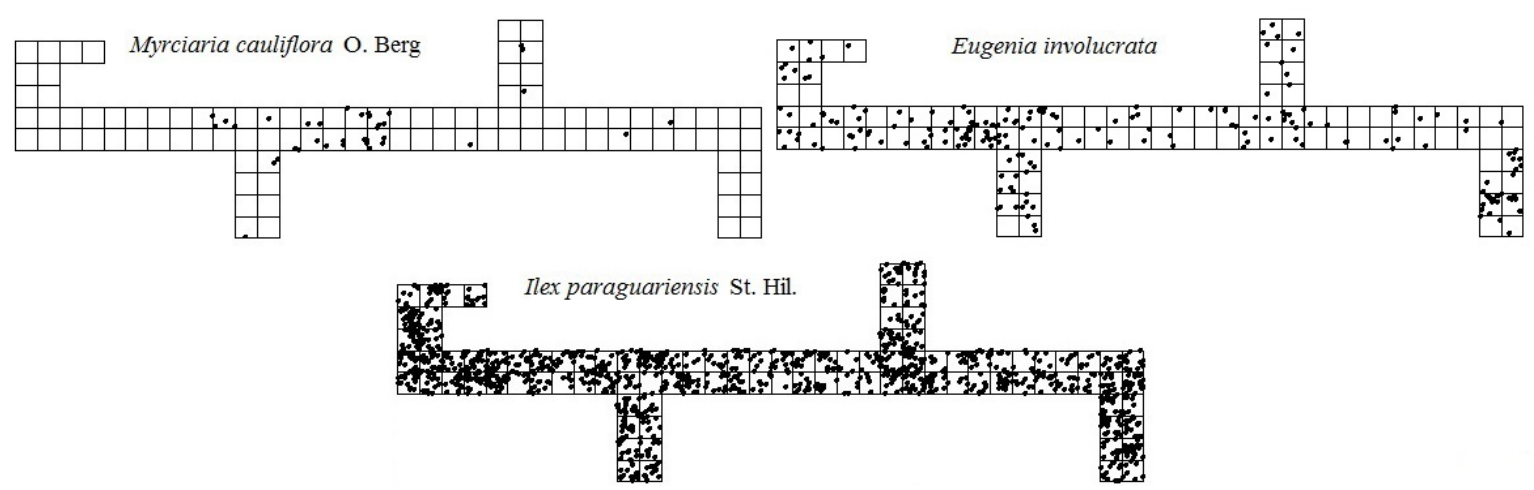

Figura 2. Disposição espacial dos indivíduos das três espécies nas parcelas permanentes.

Figure 2. Spatial disposition of individuals of tree species in permanent plots.

A espécie Eugenia involucrata, quanto à disposição dos indivíduos (Figura 2), apresenta um perfil visual mais uniforme do que agregado, porém o resultado advindo do índice de Morisita a classifica também como agregada (Tabela 2). Tonetto et al. (2013) estudaram a dinâmica populacional e produção de sementes dessa espécie em Floresta Estacional Subtropical, constatando um perfil agregado na disposição dos indivíduos e uma distribuição diamétrica exponencial negativa da população, ou seja, igualmente aos resultados de classificação da espécie da Floresta Ombrófila Mista. 
Um planejamento estratégico de manejo se torna rápido e eficaz a partir do conhecimento do comportamento espacial na disposição dos indivíduos (Figura 2), conforme se pode prever para a espécie Eugenia involucrata, a qual pode ser explorada através de duas "frentes de trabalho", uma iniciando pelo bloco 25 e caminhando em direção ao centro dessa área florestal, enquanto a outra pelo bloco 19 e seguindo a ordem inversa à anterior (Figura 1).

A espécie Ilex paraguariensis apresenta grande quantidade de indivíduos distribuídos em toda a área amostral, resultando em uma configuração visualmente mais agregada do que uniforme (Figura 2). Esse processo, oriundo da espacialização da espécie mostra que a colheita de erva-mate é viável, considerando a expressiva quantidade de seus indivíduos.

Informações relevantes adicionais, a partir da disposição espacial dos indivíduos, podem revelar realidades favoráveis para a valorização da área, ecológica ou economicamente, e planejamento de operações de manejo florestal, além do conhecimento mais detalhado do comportamento das espécies.

Os resultados da tabela 2 , em relação às informações da figura 2 , mostram a dificuldade para a classificação da dispersão espacial das espécies quando se aplica apenas um instrumento metodológico, tendo ficado evidente que somente os dados do número de indivíduos da mesma espécie nas parcelas e/ou do número de parcelas com indivíduos da mesma espécie não são variáveis determinantes na indicação do nível de agregação.

Da mesma maneira, a utilização única da técnica da espacialização das espécies (Figura 2), devido à sua semelhança na disposição espacial dos indivíduos, não evidenciaria qual delas é a mais ou a menos agregada, uniforme ou aleatória.

O ajuste das funçães de distribuição das três espécies ocorre por meio da estimação dos parâmetros pelo método da máxima verossimilhança. Desses, percebem-se algumas particularidades, como, por exemplo, os valores estimados para (c), (A) e ( $\alpha$ ), das distribuições Weibull, Beta e Gama, respectivamente, da espécie Myrciaria cauliflora, os quais são maiores em comparação com os outros parâmetros estimados, causando mudança nas curvas, que no caso dessa espécie se aproxima da curva normal (Figura 3).

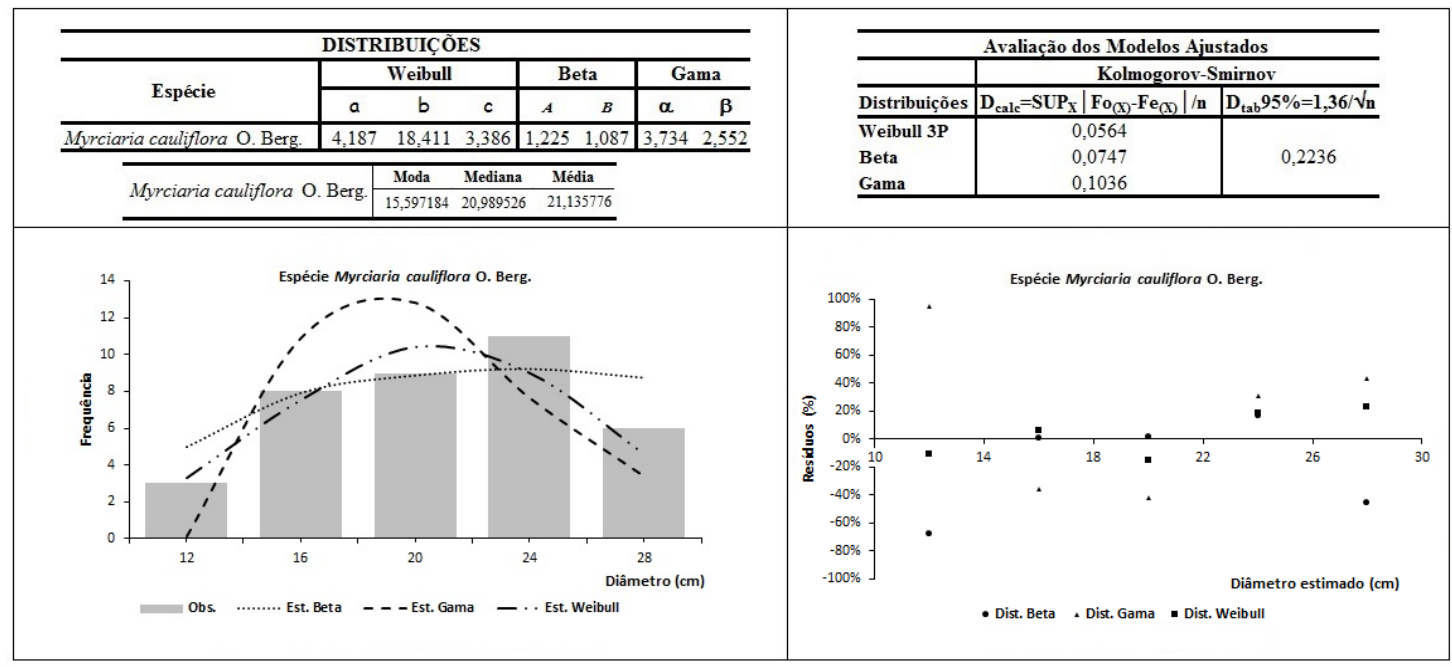

Figura 3. Resumo estatístico dos dados do ajuste da espécie Myrciaria cauliflora.

Figure 3. Statistical summary of the data of the specie Myrciaria cauliflora.

A consistência do ajuste das funções densidade de probabilidade Weibull, Beta e Gama, verificada por meio da aplicação do teste de Kolmogorov-Smirnov, comprovam que os dados observados da espécie Myrciaria cauliflora são aderentes nas três distribuições propostas ao nível de $95 \%$ de probabilidade.

Para a espécie Eugenia involucrata, a distribuição Beta não se ajustou, como aconteceu com a espécie Ilex paraguariensis para as distribuições Beta e Gama. Essa análise confirma que a distribuição de Weibull é a mais flexível para descrever distribuições diamétricas das espécies florestais (Figura 4). 


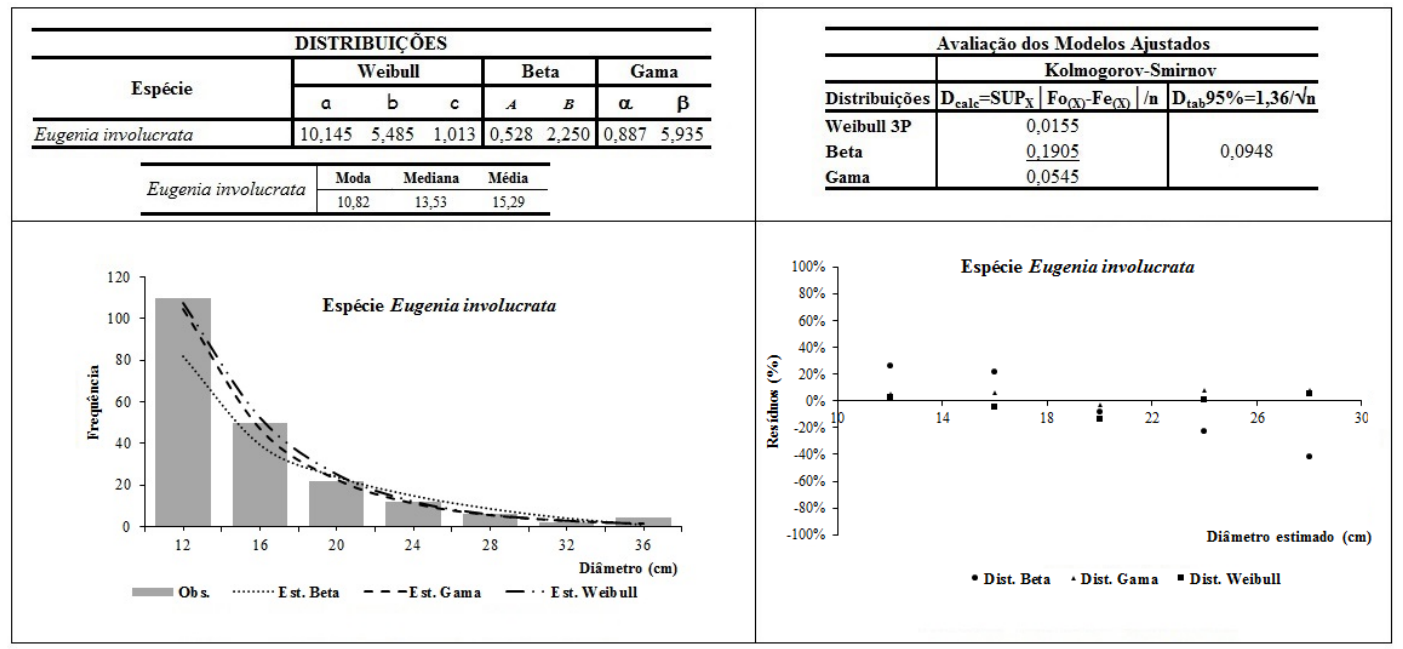

Figura 4. Resumo estatístico dos dados do ajuste da espécie Eugenia involucrata.

Figure 4. Statistical summary of the data of the specie Eugenia involucrata.

Os três gráficos contendo as curvas ajustadas aos dados observados revelam que elas seguiram um padrão similar de ajuste, apesar de as três terem sido rejeitadas pelo teste de Kolmogorov-Smirnov, conforme já citado.

A análise da distribuição gráfica dos valores residuais foi efetuada para avaliar a qualidade das estimativas, permitindo detectar discrepâncias na estimativa dos diâmetros ao longo da linha de regressão, como observado e confrontado entre a curva ajustada da distribuição Beta e o gráfico de resíduos da espécie Myrciaria cauliflora, em que se verifica uma superestimação dos diâmetros extremos (menores e maiores) em relação aos dados observados.

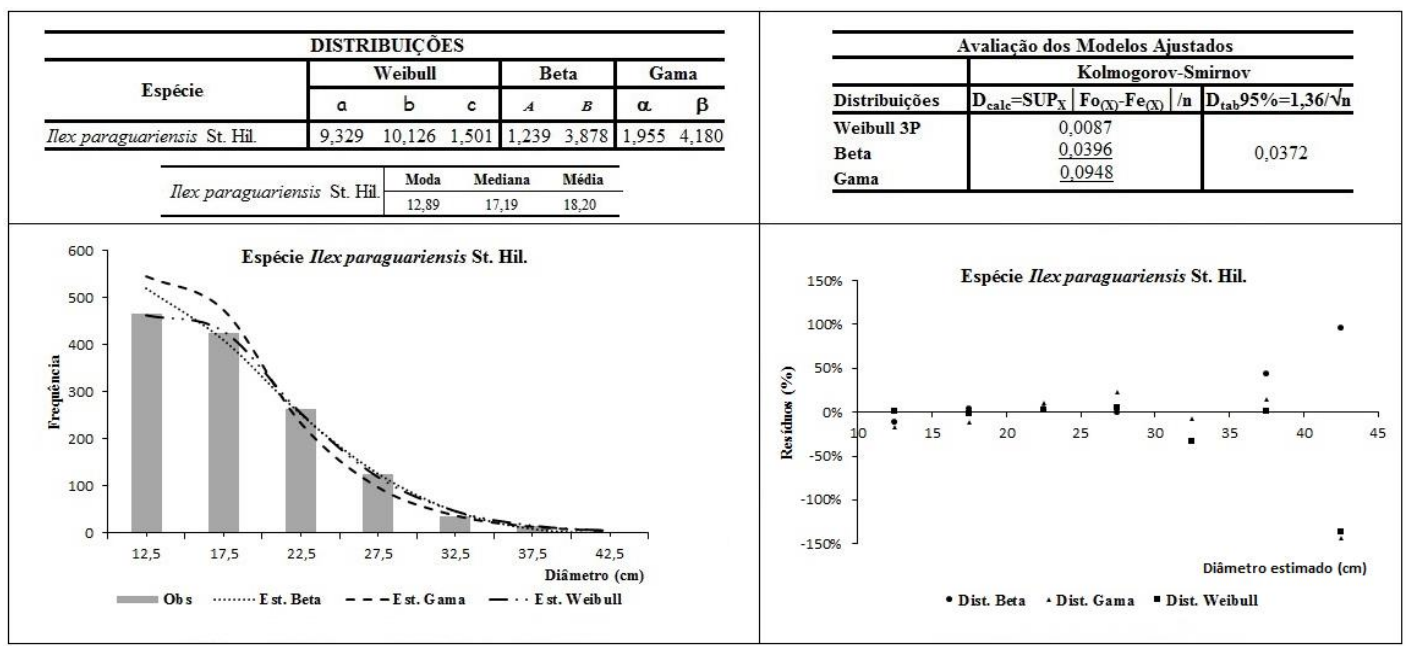

Figura 5. Resumo estatístico dos dados do ajuste da espécie Ilex paraguariensis.

Figure 5. Statistical summary of the data of the specie Ilex paraguariensis.

Os resultados dos coeficientes de determinação $\left(R^{2}\right)$ da espécie Myrciaria cauliflora para o ajuste das distribuições Weibull, Beta e Gama são $80,7 \%, 73,1 \%$ e 59,3\%, abaixo do esperado, em comparação com resultados encontrados para as espécies Eugenia involucrata $(99,7 \%, 98,7 \%$ e 99,9\%) e Ilex paraguariensis $(99,9 \%, 98,9 \%$ e $98,4 \%)$.

Esses resultados indicam as porcentagens das variações dos diâmetros observados e explicados pelos ajustes das curvas. A outra medida de precisão analisada foi o erro padrão dos resíduos, que expressa, em termos médios, a variação dos diâmetros observados da espécie Myrciaria cauliflora, da

FLORESTA, Curitiba, PR, v. 45, n. 1, p. 75 - 84, jan. / mar. 2015

Amaral, M. K. do. et al.

ISSN eletrônico 1982-4688 / ISSN impresso 0015-3826

DOI: $10.5380 /$ rf.v45i1.33679 
ordem de 7,4\%, 8,7\% e 10,7\% em relação aos diâmetros estimados pelas distribuições Weibull, Beta e Gama, respectivamente.

Analisando-se as distribuições diamétricas das populações, observa-se grande concentração de indivíduos com diâmetro inferior a $12 \mathrm{~cm}$ para Eugenia involucrata e com 17,5 cm para Ilex paraguariensis. Essa condição configura alto índice de regeneração natural em áreas de florestas com Araucaria angustifolia (Bertol) Kuntze.

A análise conjunta das curvas geradas pelas distribuições Weibull, Beta e Gama e os resultados do índice de Morisita das espécies (Tabela 2) revelam que o maior índice de agregação, oriundo da aplicação da Equação (1), gera uma curva próxima à distribuição normal, ou seja, mais simétrico. A consequência disso é um valor para a média dos diâmetros mais centralizada (Figura 3), enquanto que valores menores do índice resultam em curvas com tendência, ou seja, com assimetria à esquerda, cujo efeito se revela no valor da média dos diâmetros maior do que a mediana e a moda na distribuição diamétrica (Figuras 4 e 5).

\section{CONCLUSÕES}

- Um planejamento estratégico para o manejo e/ou conservação florestal de uma determinada área deve conter informações sobre o comportamento espacial na disposição dos indivíduos, assim como das curvas estimadas pelas distribuições diamétricas, importantes na geração de conhecimento, porque informações sobre localização dos indivíduos e das suas características comportamentais, ou da dinâmica das espécies, advindas do estudo dessa relação, permitem subsidiar melhor a análise das etapas de viabilidade do processo exploratório, ou seja, da colheita de seus frutos e de outros produtos gerados pelos seus respectivos ciclos produtivos.

\section{REFERÊNCIAS}

BAILEY, R. L.; DELL, T. R. Quantifying diameter distributions with the Weibull function. Forest Science, Lawrence, v. 19, n. 2, p. 97 - 104, 1973.

BARBOSA, J. C. A amostragem sequencial. In: Fernandes, O. A., Correia, A. C. B., De Bertoli, S. A. (FJds.). Manejo integrado de pragas e nematoides. Jaboticabal: FUNEP, p. 205 - 211, 1992.

BARRA, O. S. V.; SANQUETTA, C. R.; ARCE, J. E.; MACHADO, S. A.; CORTE, A. P. D. Proposta metodológica para ajuste ótimo da distribuição diamétrica Weibull 3P. Floresta, Curitiba, v. 34, n. 3, p. 387 - 393, 2004.

CARELLI NETTO, C.; SCHNEIDER, P. R. Distribuição diamétrica para povoamento de Pinus taeda em função da idade. In: SIMPÓSIO LATINO-AMERICANO SOBRE MANEJO FLORESTAL, 4, 2008, Santa Maria. Anais... Santa Maria, RS: Universidade Federal de Santa Maria, 2008.

CLUTTER, J. L.; BENNETT, F. A. Diameter distributions in old-field slash pine plantations. Georgia Forest Research Council Report, Atlanta, v. 13, p. 1 - 9, 1965.

DISPERATI, A. A. Biodiversidade e sustentabilidade da Floresta Ombrófila Mista na Flona de Irati, PR. Irati: UNICENTRO, 2002. 76 p. (Relatório final do projeto do CNPq: Fomento à Pesquisa/Apoio a Projeto de Pesquisa/Edital CNPq - 01/2000 - Faixa B).

DISPERATI, A. A.; OLIVEIRA FILHO, P. C. Copas de Araucária observadas em fotografias aéreas $70 \mathrm{~mm}$ colorido normal em escala muito grande (1:1.000). Ambiência, Guarapuava, PR, v. 2, p. 137 - 149, 2006.

DONADIO, L. C. Jabuticaba (Myrciaria jaboticaba (Vell.) Berg). Jaboticabal: FUNEP, 2000, 55 p.

FARIAS, P. R. S.; BARBOSA, J. C.; BUSOLI, A. C. Distribuição espacial da lagarta-do-cartucho, Spodoptera frugiperda (J. E. Smith) (Lepidoptera: Noctuidae), na cultura do milho. Neotropical Entomology, Londrina, v. 30, n. 4, p. 681 - 690, 2001.

LÖETSCH, F.; ZOERER, F.; HALLER, K. E. Forest inventory, München: BLV Verlagsgesellschaft, 1973. v. 2, 70 p. 
MACCARI JÚNIOR, A. Produtos alternativos e desenvolvimento da tecnologia industrial na cadeia produtiva da erva-mate. Curitiba: Câmara Setorial de Cadeia Produtiva da Erva-mate, 2000. 160 p. (Série PDACT, 1).

MACHADO, S. A.; AUGUSTYNCZIK, A. L. D.; FIGURA, M. A.; TÉO, S. J.; SILVA, L. C. R.; MIGUEL, E. P.; NASCIMENTO, R. G. M. Distribuição diamétrica de Araucaria angustifolia (Bert.) O. Ktze. em um fragmento de Floresta Ombrófila Mista. Scientia Agraria, Curitiba, v. 10, n. 2, p. 103 - 110, 2009.

MARUYAMA, W. I.; BARBOSA, J. C.; TOSCANO, L. C. Distribuição espacial de Oncometopia facialis (Signoret) (Hemiptera: Cicadellidae) em pomar cítrico. Neotropical Entomology. Londrina, v. 35, n. 1, 2006.

MORISITA, M. I. Index, a measure of dispersion of individuals. Researches on Population Ecology, Fukuoka, v. 4, p. 1 - 7, 1962.

NASCIMENTO, J. E. Distribuição espacial e plano de amostragem sequencial para o percevejo pequeno Piezodorus guildinii (Westwood, 1837) na cultura da soja. $137 \mathrm{f}$. Dissertação (Mestrado em Agronomia), Universidade Estadual Paulista, Jaboticabal. 1995.

NELSON, T. C. Diameter distribution and growth of loblolly pine. Forest Science. Washington, v. 10, n. 1 , p. 105 - 114. 1964.

ORELLANA, E. Funções densidade de probabilidade no ajuste da distribuição diamétrica de um fragmento de Floresta Ombrófila Mista. 122 f. Dissertação (Mestrado em Ciências Florestais) Universidade Estadual do Centro-Oeste, UNICENTRO, Irati, PR, 2009.

ORO, P.; SCHULZ, D. G.; VOLKWEIS, C. R.; BANDEIRA, K. B.; MALAVASI, C. U.; MALAVASI, M. M. Maturação fisiológica de sementes de Eugenia pyriformis Cambess e Eugenia involucrata DC. Biotemas, Florianopolis, v. 25, n. 3, p. 11 - 18, 2012.

PETERS, C. M. Sustainable harvest of non-timber plant resources in tropical moist forest: an Ecological Primer. Biodiversity support program, Washington. 1994.

PUKKALA, T. Effect of spatial distribution of trees on the volume increment of a young Scots pine stand. Siva Fennica, Helsinki, v. 2, n. 1, p. 1 - 17, 1988.

PUKKALA, T. Prediction of tree diameter and height in a Scots pine stand as a function of the spatial pattern of trees. Silva Fennica, Helsinki, v. 23, n. 2, p. 101 - 116, 1989.

RODE, R. Avaliação florística e estrutural de uma Floresta Ombrófila Mista e de uma vegetação arbórea estabelecida sob um povoamento de Araucaria angustifolia de 60 anos. 159 f. Dissertação (Mestrado em Engenharia Florestal) - Setor de Ciências Agrárias, Universidade Federal do Paraná, Curitiba, 2008.

RODE, R.; FIGUEIREDO FILHO, A.; MACHADO, S. A.; GALVÃO, F. Análise do padrão espacial de espécies e de grupos florísticos estabelecidos em um povoamento de Araucaria angustifolia e em uma Floresta Ombrófila Mista no centro-sul do Paraná. Floresta, Curitiba, v. 40, n. 2, p. 255 - 268, 2010.

SASSO, A. R. Propagação vegetativa de jabuticabeira. 64 f. 2009. Dissertação (Mestrado em Agronomia) - Universidade Tecnológica Federal do Paraná, UTFPR, Pato Branco, PR.

SOUZA, V. L.; SILVA, O. A. Estrutura e distribuição espacial de uma população de Stryphnodendron adstringens (Mart.) Coville em cerrado da Reserva Biológica e Estação Experimental de Mogi-Guaçu, estado de São Paulo, Brasil. Holos Environment, Rio Claro, SP, v. 6, p. 55 - 69, 2006.

TONETTO, T. da S.; PRADO, A. P. do; ARAÚJO, M. M.; SCCOTI, M. S. V.; FRANCO, E. T. H. Dinâmica populacional e produção de sementes de Eugenia involucrata na Floresta Estacional Subtropical. Floresta e Ambiente, Rio de Janeiro, v. 20, n. 1, p. 62 - 69, 2013.

WENDT, S. N.; SOUSA, V. A.; QUOIRIN, M. G.; SEBBENN, A. M.; MAZZA, M. C.; STURION, J. A. Caracterização genética de procedências e progênies de Ilex paraguariensis St.-Hil., utilizando marcadores RAPD. Scientia Forestalis, Piracicaba, v. 73, p. 47 - 53, 2007.

FLORESTA, Curitiba, PR, v. 45, n. 1, p. 75 - 84, jan. / mar. 2015.

Amaral, M. K. do. et al.

ISSN eletrônico 1982-4688 / ISSN impresso 0015-3826

83

DOI: $10.5380 /$ rf.v45i1.33679 
FLORESTA, Curitiba, PR, v. 45, n. 1, p. 75 - 84, jan. / mar. 2015. Amaral, M. K. do. et al. 